IJMMS 30:1 (2002) 57-62

PII. S0161171202011699

http://ijmms.hindawi.com

(c) Hindawi Publishing Corp.

\title{
MONOTONE-ITERATIVE TECHNIQUE OF LAKSHMIKANTHAM FOR THE INITIAL VALUE PROBLEM FOR A DIFFERENTIAL EQUATION WITH A STEP FUNCTION
}

\author{
S. G. HRISTOVA and M. A. RANGELOVA
}

Received 28 January 2001 and in revised form 18 June 2001

\begin{abstract}
The initial value problem for a special kind of differential equations with a step function is studied. The monotone-iterative technique of Lakshmikantham for approximate finding of the solutions of the given problem is well grounded.
\end{abstract}

2000 Mathematics Subject Classification: 34E05, 34A45.

1. Introduction. Many evolutionary processes can be described with the help of differential equations. At the same time, the solutions of a small number of linear differential equations can be found as well-known functions. That is why it is necessary to prove some approximate methods for solving different kinds of differential equations. One of the most practically used methods is the monotone-iterative technique of Lakshmikantham [1, 2, 3].

In this note, this method is well grounded for a special type of differential equations. We studied the case when the right part of the equation depends on a piecewise constant function. We note that some qualitative properties of the solutions of differential equations with a piecewise constant function (DEPCF) such as uniqueness, oscillation, and periodicity are investigated in [4]. Research in this direction is motivated by the fact that DEPCF represent a hybrid of continuous and discrete dynamical systems and combine the properties of both differential and difference equations.

2. Preliminary notes and definitions. Let $T>0$ and $0=t_{0}<t_{1}<t_{2}<\cdots<t_{p}<$ $t_{p+1}=T$ be fixed numbers.

Definition 2.1. The function $g(t):[0, T] \rightarrow \mathbb{R}$ is called a step function if $g(t)=g_{n}$ for $t_{n} \leq t<t_{n+1}$ where $g_{n}=$ const, $n=0,1, \ldots, p$.

Consider the initial value problem (IVP) for the differential equation with a step function

$$
x^{\prime}=f(x(t), x(g(t))) \text { for } t \in[0, T], \quad x(0)=c_{0},
$$

where $x \in \mathbb{R}, f: \mathbb{R} \times \mathbb{R} \rightarrow \mathbb{R}, c_{0}$ is an arbitrary constant, $g(t)$ is a step function.

We denote by $P C^{1}([0, T], \mathbb{R})$ the set of all functions $u \in C([0, T], \mathbb{R})$ for which the derivative $u^{\prime}(t)$ exists and is piecewise continuous in $[0, T]$ with points of discontinuity of first kind at the points $t_{n}, n=1,2, \ldots, p, u^{\prime}\left(t_{n}\right)=u^{\prime}\left(t_{n}+0\right)$. 
DEFINITION 2.2. The function $x(t)$ is a solution of the IVP (2.1) in the interval $[0, T]$ if the following conditions are fulfilled:

(1) $x(t) \in P C^{1}([0, T], \mathbb{R})$.

(2) The function $x(t)$ turns (2.1) into identities for $t \in[0, T]$.

DefinItion 2.3. The function $v(t) \in P C^{1}([0, T], \mathbb{R})$ is called a lower (upper) solution of the IVP (2.1) if

$$
v^{\prime}(t) \leq(\geq) f(v(t), v(g(t))), \quad v(0) \leq(\geq) c_{0} .
$$

Definition 2.4. The function $u(t)$ is called a minimal (maximal) solution of the IVP (2.1) if it is a solution of the IVP (2.1) and, for any other solution $x(t)$ of the IVP (2.1), the inequality $u(t) \leq(\geq) x(t)$ holds.

LEMMA 2.5. Let the following conditions be satisfied:

(1) the function $g(t):[0, T] \rightarrow \mathbb{R}$ is a step one and $0 \leq g(t) \leq t_{n}$ for $t \in\left[t_{n}, t_{n+1}\right)$, $n=0,1, \ldots, p$

(2) $M$ and $N$ are positive constants such that $(M+N) T \leq 1$;

(3) the function $p(t) \in P C^{1}([0, T], \mathbb{R})$ satisfies the inequalities

$$
p^{\prime}(t) \geq-M p(t)-N p(g(t)) \quad \text { for } t \in[0, T], \quad p(0) \geq 0 .
$$

Then $p(t) \geq 0$ for $t \in[0, T]$.

\section{ProOF}

CASE 1. Let $p(0)>0$. Suppose that there exists a point $t \in(0, T]$ such that $p(t)<0$. And let

$$
\xi=\inf \{t \in[0, T]: p(t) \leq 0\} .
$$

Then $\xi \in(0, T]$.

We consider the following two cases.

CASE 1.1. Let $\xi \neq T$. Denote $\lambda=\max _{0 \leq t \leq \xi} p(t), \lambda>0$. Then there exists a point $\eta \in[0, \xi)$ such that $p(\eta)=\lambda$. It follows from the mean value theorem that there exists $\xi_{0} \in(\eta, \xi)$ for which $p(\xi)-p(\eta)=p^{\prime}\left(\xi_{0}\right)(\xi-\eta)$. On the other hand, $p(\xi)-p(\eta) \leq$ $0-\lambda=-\lambda=\lambda_{1}<0$. Then

$$
\lambda_{1} \geq p^{\prime}\left(\xi_{0}\right)(\xi-\eta) .
$$

It follows from condition (3) of Lemma 2.5 that $p^{\prime}\left(\xi_{0}\right) \geq-M p\left(\xi_{0}\right)-N p\left(g\left(\xi_{0}\right)\right)$. Since $g\left(\xi_{0}\right) \leq \xi_{0}<\xi$, the inequalities $p\left(\xi_{0}\right) \leq \lambda, p\left(g\left(\xi_{0}\right)\right) \leq \lambda$ hold. Then

$$
-M p\left(\xi_{0}\right)-N p\left(g\left(\xi_{0}\right)\right) \geq \lambda_{1}(M+N) .
$$

It follows from inequalities (2.5) and (2.6) that $\lambda_{1} \geq \lambda_{1}(M+N)(\xi-\eta)$ which is equivalent to $1 \leq(M+N)(\xi-\eta)$. Since $(\xi-\eta)<T$, the inequality $1<(M+N) T$ holds. The last inequality contradicts condition (2) of Lemma 2.5. Therefore, the inequality $p(t)>0$ holds for $t \in[0, T]$.

CASE 1.2. Let $\xi=T$. Then $p(t)>0$ for $t \in[0, T)$ and $p(T)=0$, that is, $p(t) \geq 0$ for $t \in[0, T]$. 
CASE 2. Let $p(0)=0$. Suppose there exists a point $t \in(0, T]$ such that $p(t)<0$. And let

$$
\zeta=\sup \{t \in[0, T]: p(s)=0 \text { for } s \in[0, t]\} .
$$

We consider the following two cases.

CASE 2.1. Let $\zeta=0$.

CASE 2.1.1. There exists a point $\tau>0$ for which $p(t)>0$ for $t \in(0, \tau]$. If we consider the point $\tau$ instead of the point 0 and follow the proof of Case 1, we get $p(t) \geq 0$ for $t \in[\tau, T]$, that is, $p(t) \geq 0$ for $t \in[0, T]$.

CASE 2.1.2. There exists a point $\tau \in\left(0, t_{1}\right)$ such that $p(\tau)<0, p^{\prime}(\tau)<0$. According to condition (3) of Lemma 2.5 , the inequality $p^{\prime}(\tau) \geq-M p(\tau)-N p(g(\tau))$ holds. From condition (1) of Lemma 2.5 and the inequality $\tau<t_{1}$, it follows that $g(\tau)=g_{0}=0$, that is, $p(g(\tau))=0$. Then $p^{\prime}(\tau) \geq-M p(\tau)>0$ which leads to a contradiction. Hence the inequality $p(t) \geq 0$ holds for $t \in[0, T]$.

CASE 2.2. Let $\zeta>0$. If we consider the point $\zeta$ instead of the point 0 and follow the proof of Case 2.1, we get $p(t) \geq 0$ for $t \in[\tau, T]$, that is, $p(t) \geq 0$ for $t \in[0, T]$.

Consider the initial value problem for the linear differential equation with a step function

$$
x^{\prime}(t)=a x(t)+b x(g(t)), \quad x(0)=c_{0},
$$

where $a, b, c_{0}$ are constants.

LEMMA 2.6. Let $a, b, c_{0}$ be constants and the function $g(t):[0, T] \rightarrow \mathbb{R}$ be a step one such that $0 \leq g_{n} \leq t_{n}$ for $t \in\left[t_{n}, t_{n+1}\right), n=0,1, \ldots, p$. Then the initial value problem for the linear differential equation (2.8) has a unique solution for $t \in[0, T]$.

The proof of Lemma 2.6 is trivial. From Lemma 2.6, the validity of the following result follows.

COROLlary 2.7. Let $c_{0}=0$, then the IVP (2.8) has a unique solution $x(t)=0$ for $t \in[0, T]$.

Consider the IVP

$$
x^{\prime}(t)=a x(t)+b x(g(t))+f(t, g(t)), \quad x(0)=c_{0},
$$

where $a, b, c_{0}$ are constants, $f:[0, T] \times[0, T] \rightarrow \mathbb{R}$.

THEOREM 2.8. Let the function $f \in C([0, T] \times[0, T], \mathbb{R})$ and the function $g(t)$ be a step one such that $0 \leq g(t) \leq t_{n}$ for $t \in\left[t_{n}, t_{n+1}\right), n=0,1, \ldots, p$. Then the initial value problem for the linear differential equation (2.9) has a unique solution for $t \in[0, T]$.

\section{PROOF}

CASE 1. Let $a \neq 0$.

Let $t \in\left[t_{0}, t_{1}\right)$. Consider the IVP

$$
x^{\prime}(t)=a x(t)+b s_{0}+f(t, 0), \quad x(0)=c_{0},
$$

where $s_{0}=x\left(g_{0}\right)=c_{0}$. 
The solution of the IVP (2.10) exists for $t \geq 0$ and satisfies the equality

$$
x_{0}(t)=e^{a t}\left(\int_{0}^{t} e^{-a \tau} f(\tau, 0) d \tau+c_{0}\right)+\left(e^{a t}-1\right) b a^{-1} c_{0} .
$$

Let $t \in\left[t_{1}, t_{2}\right)$. Consider the IVP

$$
x^{\prime}(t)=a x(t)+b s_{1}+f\left(t, g_{1}\right), \quad x\left(t_{1}\right)=c_{1},
$$

where $s_{1}=x\left(g_{1}\right)=x_{0}\left(g_{1}\right), c_{1}=x_{0}\left(t_{1}\right)$. The solution of the IVP (2.12) exists for $t \geq t_{1}$ and satisfies the equality

$$
x_{1}(t)=e^{a\left(t-t_{1}\right)}\left(x_{0}\left(t_{1}\right)+\int_{t_{1}}^{t} e^{-a\left(\tau-t_{1}\right)} f\left(\tau, g_{1}\right) d \tau\right)+\left(e^{a\left(t-t_{1}\right)}-1\right) b a^{-1} x_{0}\left(g_{1}\right) .
$$

Let $t \in\left[t_{2}, t_{3}\right)$. Consider the IVP

$$
x^{\prime}(t)=a x(t)+b s_{2}+f\left(t, g_{2}\right), \quad x\left(t_{2}\right)=c_{2},
$$

where $s_{2}=x\left(g_{2}\right), c_{2}=x_{1}\left(t_{2}\right)$. Since $g_{2} \leq t_{2}$, then $s_{2}=x_{k}\left(g_{2}\right)$ where

$$
k= \begin{cases}0 & \text { for } g_{2} \in\left[0, t_{1}\right], \\ 1 & \text { for } g_{2} \in\left(t_{1}, t_{2}\right] .\end{cases}
$$

The solution of the IVP (2.14) exists for $t \geq t_{1}$ and satisfies the equality

$$
x_{2}(t)=e^{a\left(t-t_{2}\right)}\left(x_{1}\left(t_{2}\right)+\int_{t_{2}}^{t} e^{-a\left(\tau-t_{2}\right)} f\left(\tau, g_{2}\right) d \tau\right)+\left(e^{a\left(t-t_{2}\right)}-1\right) b a^{-1} x_{k}\left(g_{2}\right) .
$$

With the help of the solution $x_{n-1}(t)$ in the interval $\left[t_{n-1}, t_{n}\right)$ and the steps method, we construct a solution $x_{n}(t)$ of the IVP

$$
x^{\prime}(t)=a x(t)+b s_{n}+f\left(t, g_{n}\right), \quad x\left(t_{n}\right)=c_{n} \quad \text { for } t \in\left[t_{n}, t_{n+1}\right),
$$

where $s_{n}=x_{n-i}\left(g_{n}\right), c_{n}=x_{n-1}\left(t_{n}\right)$ and $i \leq n$. The solution of the IVP (2.17) exists for $t \geq t_{n}$ and satisfies the equality

$$
x_{n}(t)=e^{a\left(t-t_{n}\right)}\left(x_{n-1}\left(t_{n}\right)+\int_{t_{n}}^{t} e^{-a\left(\tau-t_{n}\right)} f\left(\tau, g_{n}\right) d \tau\right)+\left(e^{a\left(t-t_{n}\right)}-1\right) b a^{-1} x_{n-i}\left(g_{n}\right) .
$$

CASE 2. Let $a=0$. Consider the following two cases.

CASE 2.1. Let $b \neq 0$. Using the steps method, we construct the functions $x_{n}(t)$, $t \in\left[t_{n}, t_{n+1}\right), n=0,1,2, \ldots, p$ as solutions of the IVP

$$
x^{\prime}(t)=b s_{n}+f\left(t, g_{n}\right), \quad x\left(t_{n}\right)=c_{n},
$$

where $s_{n}=x_{n-i}\left(g_{n}\right), c_{n}=x_{n-1}\left(t_{n}\right)$ for $0<n \leq p$ and $i \leq n$.

Therefore,

$$
x_{n}(t)=\int_{t_{n}}^{t} f\left(\tau, g_{n}\right) d \tau+b x_{n-i}\left(g_{n}\right)\left(t-t_{n}\right)+x_{n-1}\left(t_{n}\right) .
$$


CASE 2.2. Let $b=0$. Using the steps method, we construct the functions $x_{n}(t), t \in$ $\begin{array}{llll}{\left[t_{n}, t_{n+1}\right),} & n & = & 0,1 \text {, }\end{array}$ $2, \ldots, p$ as solutions of the IVP

$$
x^{\prime}(t)=f\left(t, g_{n}\right), \quad x\left(t_{n}\right)=c_{n},
$$

where $c_{n}=x_{n-1}\left(t_{n}\right)$.

Therefore,

$$
x_{n}(t)=\int_{t_{n}}^{t} f\left(\tau, g_{n}\right) d \tau+x_{n-1}\left(t_{n}\right) \text {. }
$$

Define the function

$$
x(t)= \begin{cases}x_{0}(t) & \text { for } t \in\left[0, t_{1}\right), \\ x_{1}(t) & \text { for } t \in\left[t_{1}, t_{2}\right), \\ \vdots & \\ x_{p}(t) & \text { for } t \in\left[t_{p}, t_{p+1}\right] .\end{cases}
$$

The function $x(t)$ is a solution of the IVP (2.9) in $[0, T]$. Suppose there exist two different solutions $x(t)$ and $y(t)$ of the IVP (2.9). Define the function $q(t)=x(t)-y(t)$, $t \in[0, T]$. The function $q(t)$ satisfies the IVP (2.8), where $c_{0}=0$. By the Corollary 2.7, it follows that $q(t)=0$ for $t \in[0, T]$. Therefore the IVP (2.9) has a unique solution.

3. Main results. We will apply the monotone-iterative technique to find an approximate solution of the initial value problem for a nonlinear differential equation with a step function.

THEOREM 3.1. Let the following conditions be fulfilled:

(1) the function $g(t) \in([0, T], \mathbb{R})$ is a step one such that $0 \leq g(t) \leq t_{n}$ for $t \in$ $\left[t_{n}, t_{n+1}\right), n=0,1, \ldots, p$;

(2) $M$ and $N$ are positive constants such that $(M+N) T \leq 1$;

(3) the function $f \in C\left(\mathbb{R}^{2}, \mathbb{R}\right)$ and for $x_{1} \geq x_{2}, y_{1} \geq y_{2}$, the inequality

$$
f\left(x_{1}, y_{1}\right)-f\left(x_{2}, y_{2}\right) \geq-M\left(x_{1}-x_{2}\right)-N\left(y_{1}-y_{2}\right)
$$

holds;

(4) the functions $v_{0}(t)$ and $w_{0}(t)$ are lower and upper solutions of the IVP (2.1) and $v_{0}(t) \leq w_{0}(t)$ for $t \in[0, T]$.

Then there exist two sequences of functions $\left\{v_{n}(t)\right\}_{0}^{\infty}$ and $\left\{w_{n}(t)\right\}_{0}^{\infty}$ such that

(a) the sequences are increasing and decreasing, respectively;

(b) the functions $v_{n}(t), w_{n}(t)$ are lower and upper solutions of the IVP (2.1);

(c) the sequences are uniformly convergent in the interval $[0, T]$;

(d) the limits $v(t)=\lim _{n \rightarrow \infty} v_{n}(t), w(t)=\lim _{n \rightarrow \infty} w_{n}(t)$ are minimal and maximal solutions of the IVP (2.1), respectively. 
Proof. Let the function $\eta(t) \in C([0, T], \mathbb{R}), v_{0}(t) \leq \eta(t) \leq w_{0}(t)$, be fixed. Consider the initial value problem for the linear differential equation with a step function

$$
\begin{gathered}
x^{\prime}(t)=f(\eta(t), \eta(g(t)))-M(x(t)-\eta(t))-N(x(g(t))-\eta(g(t))), \\
x(0)=c_{0} .
\end{gathered}
$$

By Theorem 2.8, the IVP (3.2) has a unique solution $x(t)$ for $t \in[0, T]$. Define the mapping $A$ by the equality $A \eta(t)=x(t)$ where $x(t)$ is the unique solution of the IVP (3.2). We prove that the operator $A$ satisfies the following properties:

(i) $v_{0}(t) \leq A v_{0}(t), w_{0}(t) \geq A w_{0}(t)$;

(ii) for any function $u_{1}(t), u_{2}(t) \in P C^{1}([0, T]$, $\mathbb{R})$ such that $v_{0}(t) \leq u_{1}(t) \leq u_{2}(t)$ $\leq w_{0}(t)$, the inequality $A u_{1}(t) \leq A u_{2}(t)$ holds.

Indeed, let $A v_{0}(t)=v_{1}(t)$. The function $v_{1}(t)$ is continuous and it is the solution of the IVP (3.2) for $\eta(t)=v_{0}(t)$. Set $p(t)=v_{1}(t)-v_{0}(t)$. Then $p^{\prime}(t)=v_{1}^{\prime}(t)-v_{0}^{\prime}(t) \geq$ $v_{1}^{\prime}(t)-f\left(v_{0}(t), v_{0}(g(t))\right)=-M p(t)-N p(g(t))$ and $p(0)=v_{1}(0)-v_{0}(0) \geq 0$.

By Lemma 2.5 the function $p(t)$ is nonnegative in $[0, T]$, that is, $A v_{0}(t) \geq v_{0}(t)$. Let $A w_{0}(t)=w_{1}(t)$. The function $w_{1}(t)$ is continuous and it is a solution of (3.2) for $\eta(t)=w_{0}(t)$. Set $p(t)=w_{0}(t)-w_{1}(t)$. Then

$$
\begin{aligned}
p^{\prime}(t) \geq & f\left(w_{0}(t), w_{0}(g(t))\right)-f\left(w_{0}(t), w_{0}(g(t))\right) \\
& +M\left(w_{1}(t)-w_{0}(t)\right)+N\left(w_{1}(g(t))-w_{0}(g(t))\right) \\
= & -M p(t)-N p(g(t)), \quad p(0) \geq 0 .
\end{aligned}
$$

By Lemma 2.5 the function $p(t)$ is nonnegative in $[0, T]$, that is, $A w_{0}(t) \leq w_{1}(t)$. Therefore, property (i) is satisfied.

Let $u_{1}, u_{2} \in P C^{1}([0, T], \mathbb{R})$ and $v_{0}(t) \leq u_{1}(t) \leq u_{2}(t) \leq w_{0}(t)$. If $x_{1}(t)=A u_{1}(t)$ and $x_{2}(t)=A u_{2}(t)$, then the function $p(t)=x_{2}(t)-x_{1}(t)$ satisfies the equality

$$
\begin{aligned}
p^{\prime}(t) & =f\left(u_{2}(t), u_{2}(g(t))\right)-M\left(x_{2}(t)-u_{2}(t)\right)-N\left(x_{2}(g(t))-u_{2}(g(t))\right) \\
& -f\left(u_{1}(t), u_{1}(g(t))\right)+M\left(x_{1}(t)-u_{1}(t)\right)+N\left(x_{1}(g(t))-u_{1}(g(t))\right) .
\end{aligned}
$$

Due to Theorem 3.1(3), we get $p^{\prime}(t) \geq-M\left(x_{2}(t)-x_{1}(t)\right)-N\left(x_{2}(g(t))-x_{1}(g(t))\right)=$ $-M p(t)-N p(g(t))$ and $p(0)=0$. By Lemma 2.5 the inequality $p(t) \geq 0$ holds, that is, $A u_{1}(t) \leq A u_{2}(t)$. Therefore, property (ii) is satisfied.

Define the sequences $\left\{v_{n}(t)\right\}_{0}^{\infty}$ and $\left\{w_{n}(t)\right\}_{0}^{\infty}$ with the help of the equalities $v_{n}(t)=$ $A v_{n-1}(t), w_{n}(t)=A w_{n-1}(t), n \geq 1$. By the proof of Theorem 2.8 we get

$$
\begin{aligned}
v_{n}^{(0)}(t)= & e^{-M t}\left(c_{0}+\int_{0}^{t} e^{M \tau} \varphi_{n-1}^{(0)}(\tau, 0) d \tau\right) \\
& +\left(e^{-M t}-1\right) N M^{-1} c_{0} \quad \text { for } t \in\left[0, t_{1}\right), \\
v_{n}^{(m)}(t)= & e^{-M\left(t-t_{m}\right)}\left(v_{n}^{(m-1)}\left(t_{m}\right)+\int_{t_{m}}^{t} e^{M\left(\tau-t_{m}\right)} \varphi_{n-1}^{(m)}\left(\tau, g_{m}\right) d \tau\right) \\
& +\left(e^{-M\left(t-t_{m}\right)}-1\right) N M^{-1} v_{n}^{(m-i)}\left(g_{m}\right) \\
& \quad \text { for } t \in\left[t_{m}, t_{m+1}\right), m=1,2, \ldots, p, \quad i \leq m,
\end{aligned}
$$




$$
\begin{aligned}
& w_{n}^{(0)}(t)= e^{-M t}\left(c_{0}+\int_{0}^{t} e^{M \tau} \psi_{n-1}^{(0)}(\tau, 0) d \tau\right) \\
&+\left(e^{-M t}-1\right) N M^{-1} c_{0} \quad \text { for } t \in\left[0, t_{1}\right), \\
& w_{n}^{(m)}(t)= e^{-M\left(t-t_{m}\right)}\left(w_{n}^{(m-1)}\left(t_{m}\right)+\int_{t_{m}}^{t} e^{M\left(\tau-t_{m}\right)} \psi_{n-1}^{(m)}\left(\tau, g_{m}\right) d \tau\right) \\
&+\left(e^{-M\left(t-t_{m}\right)}-1\right) N M^{-1} w_{n}^{(m-i)}\left(g_{m}\right) \\
& \text { for } t \in\left[t_{m}, t_{m+1}\right), \quad m=1,2, \ldots, p, \quad i \leq m, \\
& v_{n}(t)=\left\{\begin{array}{lll}
v_{n}^{(0)}(t) & \text { for } t \in\left[0, t_{1}\right), \\
v_{n}^{(1)}(t) & \text { for } t \in\left[t_{1}, t_{2}\right), \\
\vdots & w_{n}(t)= \begin{cases}w_{n}^{(0)}(t) & \text { for } t \in\left[0, t_{1}\right), \\
w_{n}^{(1)}(t) & \text { for } t \in\left[t_{1}, t_{2}\right), \\
\vdots & \end{cases} \\
w_{n}^{(p)}(t) & \text { for } t \in\left[t_{p}, t_{p+1}\right],
\end{array}\right.
\end{aligned}
$$

where, for $m=0,1, \ldots, p$,

$$
\begin{aligned}
& \varphi_{n-1}^{(m)}\left(t, g_{m}\right)=M v_{n-1}^{(m)}(t)+N v_{n-1}^{(m)}\left(g_{m}\right)+f\left(v_{n-1}^{(m)}(t), v_{n-1}^{(m)}\left(g_{m}\right)\right), \\
& \psi_{n-1}^{(m)}\left(t, g_{m}\right)=M w_{n-1}^{(m)}(t)+N w_{n-1}^{(m)}\left(g_{m}\right)+f\left(w_{n-1}^{(m)}(t), w_{n-1}^{(m)}\left(g_{m}\right)\right) .
\end{aligned}
$$

By properties (i) and (ii) of the operator $A$, the following inequalities hold:

$$
v_{0}(t) \leq v_{1}(t) \leq \cdots \leq v_{n}(t) \leq w_{n}(t) \leq \cdots \leq w_{1}(t) \leq w_{0}(t) \text { for } t \in[0, T]
$$

The sequences $\left\{v_{n}(t)\right\}_{0}^{\infty}$ and $\left\{w_{n}(t)\right\}_{0}^{\infty}$ are equicontinuous and uniformly bounded in the intervals $\left[t_{m}, t_{m+1}\right), m=0,1, \ldots, p$. Therefore, they are uniformly convergent on $\left[t_{m}, t_{m+1}\right)$. We denote $\lim _{n \rightarrow \infty} v_{n}^{(m)}(t)=v^{(m)}(t)$ and $\lim _{n \rightarrow \infty} w_{n}^{(m)}(t)=w^{(m)}(t)$.

Taking the limit as $n \rightarrow \infty$ into equalities (3.6) and (3.8), we obtain that the functions $v^{(m)}(t)$ and $w^{(m)}(t)$ are solutions of the integral equations,

$$
\begin{aligned}
v^{(m)}(t)= & e^{-M\left(t-t_{m}\right)}\left(v^{(m-1)}\left(t_{m}\right)+\int_{t_{m}}^{t} e^{M\left(\tau-t_{m}\right)} \varphi^{(m)}\left(\tau, g_{m}\right) d \tau\right) \\
& +\left(e^{-M\left(t-t_{m}\right)}-1\right) N M^{-1} v^{(m-i)}\left(g_{m}\right) \quad \text { for } t \in\left[t_{m}, t_{m+1}\right), i \leq m, \\
w^{(m)}(t)= & e^{-M\left(t-t_{m}\right)}\left(w^{(m-1)}\left(t_{m}\right)+\int_{t_{m}}^{t} e^{M\left(\tau-t_{m}\right)} \psi^{(m)}\left(\tau, g_{m}\right) d \tau\right) \\
& +\left(e^{-M\left(t-t_{m}\right)}-1\right) N M^{-1} w^{(m-i)}\left(g_{m}\right) \quad \text { for } t \in\left[t_{m}, t_{m+1}\right), i \leq m,
\end{aligned}
$$

where, for $m=0,1, \ldots, p$,

$$
\begin{aligned}
& \varphi^{(m)}\left(t, g_{m}\right)=M v^{(m)}(t)+N v^{(m)}\left(g_{m}\right)+f\left(v^{(m)}(t), v^{(m)}\left(g_{m}\right)\right), \\
& \psi^{(m)}\left(t, g_{m}\right)=M w^{(m)}(t)+N w^{(m)}\left(g_{m}\right)+f\left(w^{(m)}(t), w^{(m)}\left(g_{m}\right)\right) .
\end{aligned}
$$


Define the functions

$$
v(t)=\left\{\begin{array}{ll}
v^{(0)}(t) & \text { for } t \in\left[0, t_{1}\right), \\
v^{(1)}(t) & \text { for } t \in\left[t_{1}, t_{2}\right), \\
\vdots & \\
v^{(p)}(t) & \text { for } t \in\left[t_{p}, t_{p+1}\right],
\end{array} \quad w(t)= \begin{cases}w^{(0)}(t) & \text { for } t \in\left[0, t_{1}\right), \\
w^{(1)}(t) & \text { for } t \in\left[t_{1}, t_{2}\right), \\
\vdots & \\
w^{(p)}(t) & \text { for } t \in\left[t_{p}, t_{p+1}\right] .\end{cases}\right.
$$

From equalities (3.12) it follows that the functions $v(t)$ and $w(t)$ are solutions of the IVP (2.1). We prove that $v(t)$ and $w(t)$ are, respectively, minimal and maximal solutions of the IVP (2.1). Let $x(t)$ be an arbitrary solution of the IVP (2.1) in $[0, T]$ such that $v_{0}(t) \leq x(t) \leq w_{0}(t)$.

Assume that $v_{n}(t) \leq x(t) \leq w_{n}(t)$ in $[0, T]$ for some $n$. Set $p(t)=x(t)-v_{n+1}(t)$. Then we get

$$
\begin{aligned}
p^{\prime}(t)= & f(x(t), x(g(t)))-f\left(v_{n}(t), v_{n}(g(t))\right)+M\left(v_{n+1}(t)-v_{n}(t)\right) \\
& +N\left(v_{n+1}(g(t))-v_{n}(g(t))\right) \\
\geq & -M p(t)-N p(g(t)) \quad \text { for } t \in[0, T], \\
p(0)= & 0 .
\end{aligned}
$$

By Lemma 2.5 the inequality $p(t) \geq 0$ holds, that is, $x(t) \geq v_{n+1}(t)$ for $t \in[0, T]$. By arguments analogous to those above, we get $x(t) \leq w_{n+1}(t)$ for $t \in[0, T]$.

By induction we obtain that $v_{n}(t) \leq x(t) \leq w_{n}(t)$ for any $n \in N \cup\{0\}$. After passing to the limit for $n \rightarrow \infty$ we get $v(t) \leq x(t) \leq w(t)$, that is, $v(t)$ is a minimal solution of the IVP (2.1) and $w(t)$ is a maximal solution of the IVP (2.1).

REMARK 3.2. If the conditions of Theorem 3.1 are fulfilled and the IVP (2.1) has a unique solution $x(t) \in[0, T]$, then there exist two sequences $\left\{v_{n}(t)\right\}_{0}^{\infty}$ and $\left\{w_{n}(t)\right\}_{0}^{\infty}$ that are uniformly convergent to the unique solution $x(t)$ in the interval $[0, T]$.

\section{REFERENCES}

[1] G. S. Ladde, V. Lakshmikantham, and A. S. Vatsala, Monotone Iterative Techniques for Nonlinear Differential Equations, Pitman, Massachusetts, 1985.

[2] V. Lakshmikantham, Monotone iterative technology for nonlinear differential equations, Differential Equations: Qualitative Theory, Vol. I, II (Sieged, 1984), Colloque. Math. Soc. Jeans Boyish, vol. 47, North-Holland publishing, Amsterdam, 1987, pp. 633647.

[3] V. Lakshmikantham and S. Leela, Existence and monotone method for periodic solutions of first-order differential equations, J. Math. Anal. Appl. 91 (1983), 237-243.

[4] J. Wiener and V. Lakshmikantham, Differential equations with piecewise constant argument and impulsive equations, Nonlinear Stud. 7 (2000), no. 1, 60-69.

S. G. Hristova: Plovdiv University, Department of Applied Mathematics, Plovdiv 4000, BULGARIA

E-mail address: snehri@hotmai1.com

M. A. RANGelova: Plovdiv University, FaCUlty of MATHEMATICS AND COMPUTER SCIENCE, PlovDiV 4000, BULGARIA

E-mail address: rmarinast@hotmai 1.com 


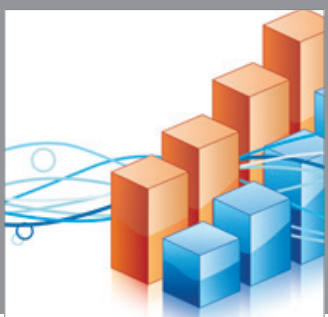

Advances in

Operations Research

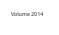

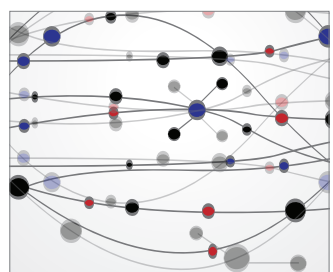

\section{The Scientific} World Journal
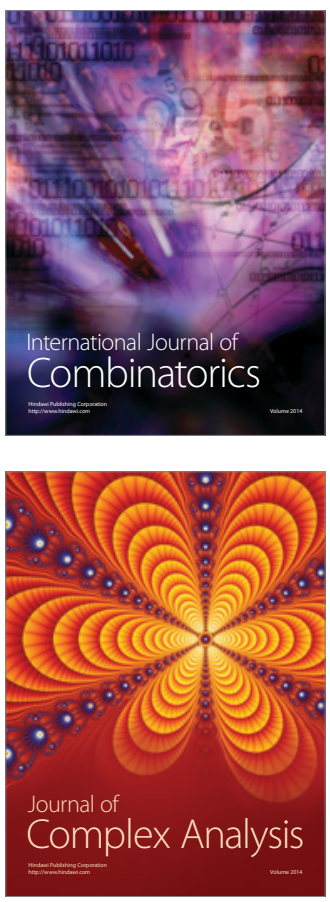

International Journal of

Mathematics and

Mathematical

Sciences
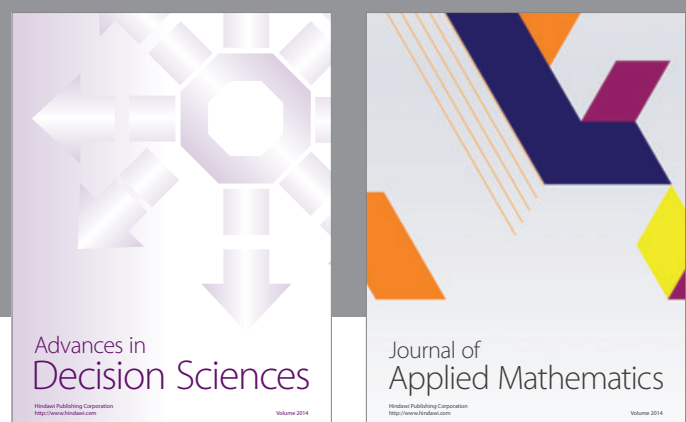

Journal of

Applied Mathematics
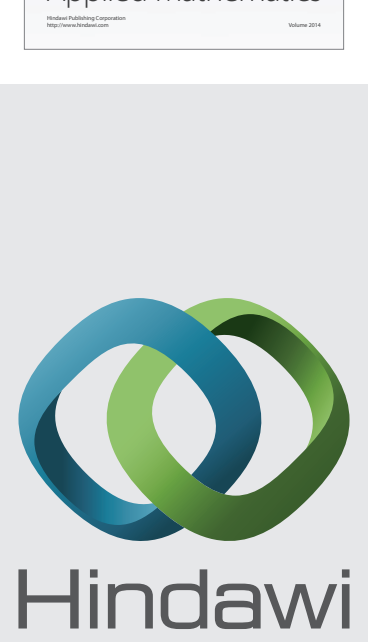

Submit your manuscripts at http://www.hindawi.com
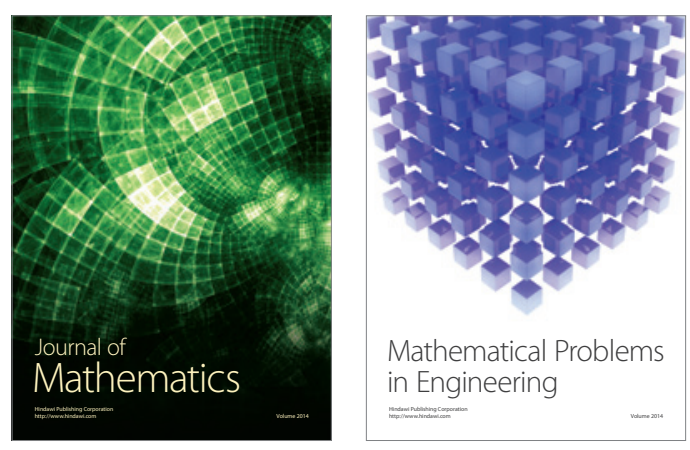

Mathematical Problems in Engineering
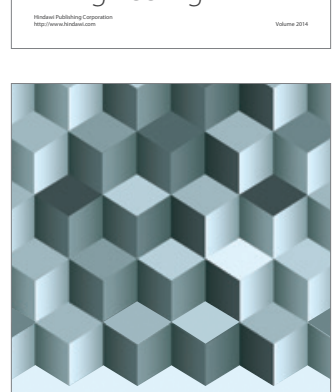

Journal of

Function Spaces
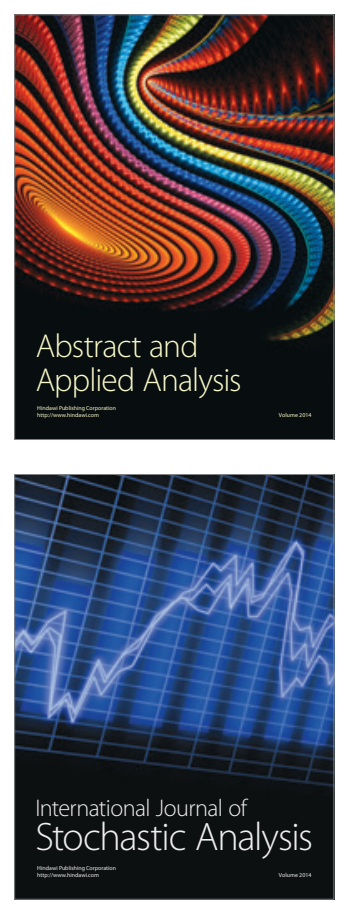

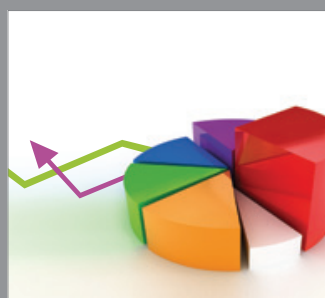

ournal of

Probability and Statistics

Promensencen
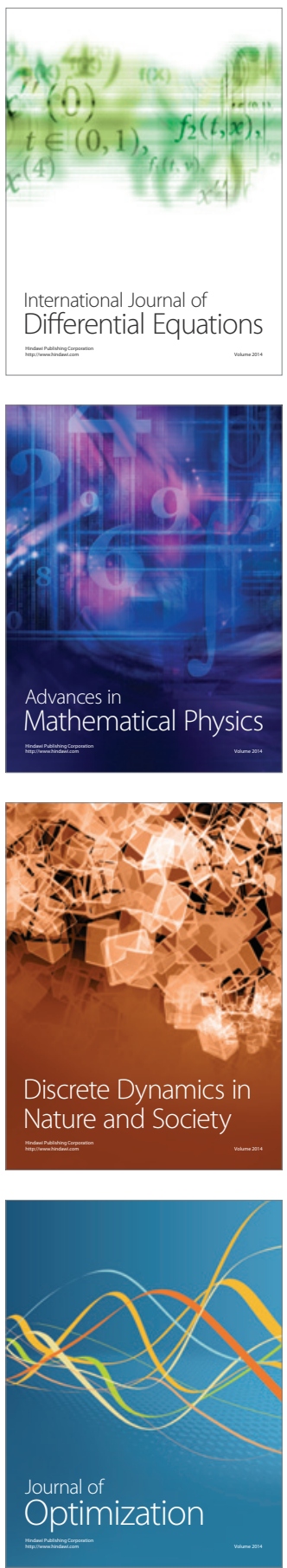